\title{
Observation of hypersonic phononic crystal effects in porous silicon superlattices
}

\author{
L. C. Parsons and G. T. Andrews ${ }^{\text {a) }}$ \\ Department of Physics and Physical Oceanography, Memorial University, St. John's, \\ Newfoundland A1B 3X7, Canada
}

(Received 30 September 2009; accepted 27 November 2009; published online 18 December 2009)

\begin{abstract}
Brillouin light scattering experiments were carried out on porous silicon superlattices with modulation wavelengths in the range 37-167 nm. Phonon frequencies deduced from the Brillouin spectra show good agreement with those obtained from an elastic continuum model for a system with one-dimensional periodicity. Evidence for the existence of a hypersonic phononic bandgap and zone-folded longitudinal acoustic phonons is reported. () 2009 American Institute of Physics. [doi:10.1063/1.3275742]
\end{abstract}

It is well known that the introduction of artificial spatial periodicity in the elastic properties of a materials system results in Brillouin zone-folding. Such folding is often accompanied by the appearance of bandgaps in the phonon frequency spectrum and thus materials systems that exhibit this phenomenon are promising candidates for phononic crystals. Porous silicon $(\pi-\mathrm{Si})$ is especially interesting in this regard since its elastic properties can be varied over nanometer length scales and because it can be readily integrated into existing silicon technologies.

In this letter, the results of Brillouin scattering experiments on $\pi$-Si superlattices (SLs) are reported. Good agreement is obtained between measured phonon frequencies and those calculated from a one-dimensional elastic continuum model. Clear evidence for the existence of an acoustic bandgap and for zone-folding of longitudinal acoustic phonons is presented.

Two sets of $\pi$-Si SLs and associated single layer films were synthesized by anodization of $p^{+}$-type (100)-oriented crystalline silicon $(5-20 \mathrm{~m} \Omega \mathrm{cm})$ in an electrolyte composed of $1(49 \% \mathrm{HF}): 1\left(\mathrm{C}_{2} \mathrm{H}_{5} \mathrm{OH}\right)$. Each set was made using a different supply of $\mathrm{HF}$ with one solution yielding single layer films with gravimetrically-determined porosities of $0.59\left(\mathrm{~J}=149 \mathrm{~mA} / \mathrm{cm}^{2}\right)$ and $0.52\left(\mathrm{~J}=101 \mathrm{~mA} / \mathrm{cm}^{2}\right)$, while the other resulted in films with porosities of 0.56 $\left(\mathrm{J}=149 \mathrm{~mA} / \mathrm{cm}^{2}\right)$ and $0.46\left(\mathrm{~J}=101 \mathrm{~mA} / \mathrm{cm}^{2}\right)$. The difference in film porosity for the same applied current density is ascribed to slight differences in HF concentration in the two solutions. The single layer films were $\sim 5 \mu \mathrm{m}$ thick. SLs with a binary periodic variation in porosity were formed by alternating the current density between the above values. Etching times were chosen so that the constituent layer thicknesses, $d_{1}$ and $d_{2}$, were nearly equal [see Fig. 1(a)]. SLs with modulation wavelengths of $D=d_{1}+d_{2}$ from $37 \mathrm{~nm}$ to $167 \mathrm{~nm}$ were made for the Brillouin experiments. The porosities, refractive indices, and elastic properties of the SL constituent layers were taken to be the same as those of single layer films formed under identical conditions. It is noted that images like that of Fig. 1(a) were not obtained for SLs with $D<500 \mathrm{~nm}$. Indirect evidence of prescribed SL formation, however, was provided by measurements of overall SL film

${ }^{a)}$ Electronic mail: tandrews@mun.ca. thickness $(\sim 5 \mu \mathrm{m})$ which was within $10 \%$ of the value calculated using known etch parameters.

Brillouin scattering experiments were carried out in air at room temperature using a backscattering geometry [see Fig. 1(b)]. Incident light of wavelength $\lambda_{i}=532 \mathrm{~nm}$ at a power of $\sim 50 \mathrm{~mW}$ was provided by a $\mathrm{Nd}: \mathrm{YVO}_{4}$ laser. The scattered light was collected with $\mathrm{f} / 2.8$ or $\mathrm{f} / 4.0$ optics, with the latter being used to prevent light specularly reflected from the sample from impinging on the active lens aperture when collecting spectra at very small incident angles. A tandem Fabry-Perot interferometer was used to frequencyanalyze the scattered light.

Brillouin spectra were collected from the single layer films in order to obtain the bulk acoustic mode velocities of the corresponding (i.e., same porosity) SL constituent layers. These were required, together with the refractive indices of the single layer films, for the calculations described below. The bulk phonon velocities were determined from spectra collected at $5^{\circ} \leq \alpha \leq 10^{\circ}$. This corresponds to internal angles between $2^{\circ}$ and $4^{\circ}$ and hence these velocities were taken as those of phonons propagating in a direction perpendicular to the film surface. The refractive index of each single layer film was obtained using the gravimetrically determined porosity and the two-component Bruggeman effective medium equation. ${ }^{1}$ These values, along with film porosities, are given in Table I. The lack of a clear trend in velocity with increasing porosity may be due to the influence of morphology, a second parameter upon which the acoustic velocities in $\pi$-Si are known to strongly depend. ${ }^{2}$ (a)

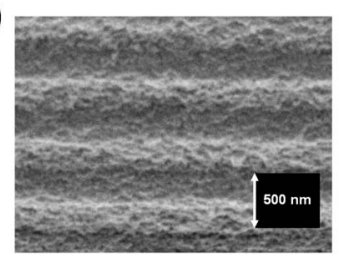

(b)

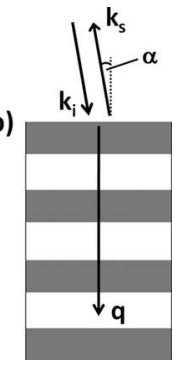

FIG. 1. (a) Cross-sectional scanning electron micrograph of a $D=500 \mathrm{~nm}$ SL. The bright (dark) regions correspond to layers with a porosity of 0.56 (0.46). (b) Scattering geometry. The wavevectors of the probed phonon and the incident (scattered) light are $\mathbf{q}$ and $\mathbf{k}_{i}\left(\mathbf{k}_{s}\right)$, respectively. 
TABLE I. Porosity, refractive index, and bulk acoustic phonon velocities for single layer porous silicon films.

\begin{tabular}{ccccc}
\hline \hline Set no. & Porosity & Refractive index & $\begin{array}{c}V_{T} \\
(\mathrm{~km} / \mathrm{s})\end{array}$ & $\begin{array}{c}V_{L} \\
(\mathrm{~km} / \mathrm{s})\end{array}$ \\
\hline \multirow{2}{*}{1} & $0.59 \pm 0.02$ & $2.1 \pm 0.1$ & $2.1 \pm 0.1$ & $4.3 \pm 0.2$ \\
& $0.52 \pm 0.03$ & $2.4 \pm 0.1$ & $2.8 \pm 0.2$ & $4.6 \pm 0.2$ \\
2 & $0.56 \pm 0.02$ & $2.2 \pm 0.1$ & $2.3 \pm 0.2$ & $4.2 \pm 0.2$ \\
& $0.46 \pm 0.02$ & $2.6 \pm 0.2$ & $2.7 \pm 0.3$ & $4.4 \pm 0.3$ \\
\hline
\end{tabular}

SL spectra were collected at $5^{\circ} \leq \alpha \leq 8^{\circ}$, yielding, by Snell's law, an internal angle range of $2^{\circ}$ to $4^{\circ}$. The probed phonon thus traveled in a direction that is very nearly perpendicular to the plane of the constituent layers. A series of Brillouin spectra collected under these conditions is shown in Fig. 2. For some of the SLs, spectra were also collected at higher $\alpha$ to check the dependence of peak frequency shift on incident angle. The shifts of all peaks observed at small $\alpha$ were found to be essentially independent of incident angle.

In a Brillouin scattering experiment conducted using a backscattering geometry, the magnitude of the probed phonon wavevector (either transverse or longitudinal) is

$$
q=2 K
$$

where $K$, for a system with a square-wave spatial periodicity in refractive index, is the photonic Bloch wavevector given by $^{3}$

$$
\begin{aligned}
K= & \frac{1}{D} \cos ^{-1}\left[\cos \left(k_{i} n_{1} d_{1}\right) \cos \left(k_{i} n_{2} d_{2}\right)\right. \\
& \left.-\frac{1}{2}\left(\frac{n_{1}}{n_{2}}+\frac{n_{2}}{n_{1}}\right) \sin \left(k_{i} n_{2} d_{2}\right) \sin \left(k_{i} n_{1} d_{1}\right)\right] .
\end{aligned}
$$

Here, $n_{1}$ and $n_{2}$ are the known constituent layer refractive indices and $k_{i}=2 \pi / \lambda_{i}$. For the $0.59 / 0.52$ and the $0.56 / 0.46$ SLs, Eq. (1) yields $q \approx 53$ and $56 \mu \mathrm{m}^{-1}$, respectively. (a)

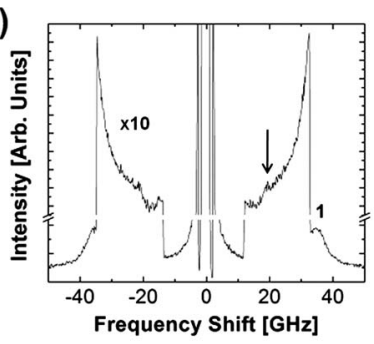

(c)

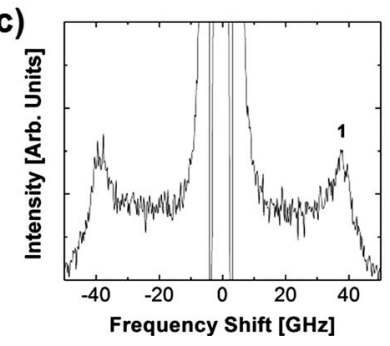

(b)

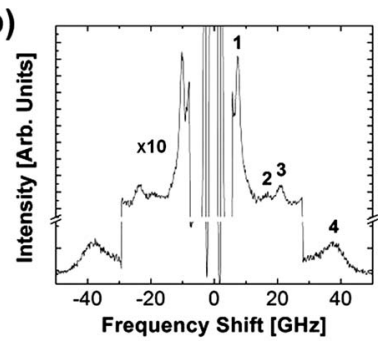

(d)

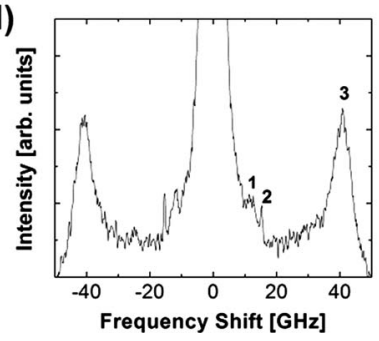

FIG. 2. Spectra collected from (a) a $D=61 \mathrm{~nm} 0.59 / 0.52$ superlattice, (b) a $D=98 \mathrm{~nm} 0.59 / 0.52$ superlattice, (c) a $D=123 \mathrm{~nm} 0.59 / 0.52$ superlattice, and (d) a $D=167 \mathrm{~nm} 0.56 / 0.46$ superlattice (smoothed using three-point averaging). The arrow indicates a weak feature that was difficult to fit to a Lorentzian and, hence, has a large uncertainty in frequency shift. This and similar features in other spectra appear in Fig. 3 and are indicated by large frequency-axis error bars.

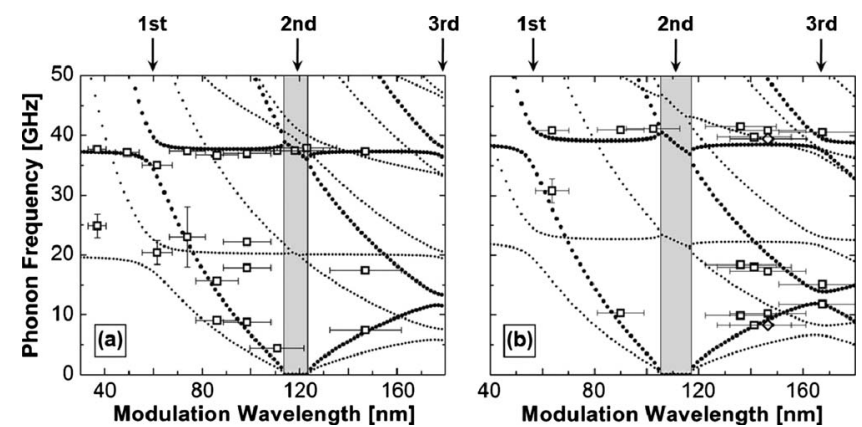

FIG. 3. Phonon frequency vs modulation wavelength $(D)$ for superlattices with constituent layer porosities of (a) 0.59 and 0.52 and (b) 0.56 and 0.46 Thick (thin) broken lines: calculated curves for the longitudinal (transverse) mode; shaded region: values of $D$ for which the probing light is in a photonic bandgap. Arrows mark the approximate values of $D$ for which $q$ coincides with the edges of the first, second, and third phononic Brillouin zones, respectively. Open symbols: experimental data; diamonds: duplicate $D=147 \mathrm{~nm}$ sample. The frequency scale on both plots is the same.

Due to the square-wave periodicity in the elastic properties, the phonon should behave as a phononic Bloch wave and therefore undergo zone-folding into the mini-Brillouin zone corresponding to $D$. The phonon then obeys the selection rule for zone-folding given by ${ }^{3}$

$$
Q=q+\frac{2 l \pi}{D},
$$

where $l$ is an integer, $Q$ is the phononic Bloch wavevector, expressed as

$$
\begin{aligned}
Q= & \frac{1}{D} \cos ^{-1}\left[\cos \left(\frac{\Omega d_{1}}{V_{1}}\right) \cos \left(\frac{\Omega d_{2}}{V_{2}}\right)\right. \\
& \left.-\frac{1}{2} F \sin \left(\frac{\Omega d_{1}}{V_{1}}\right) \sin \left(\frac{\Omega d_{2}}{V_{2}}\right)\right],
\end{aligned}
$$

and $F$ is a sample parameter given by

$$
F=\left[\frac{\rho\left(1-\zeta_{1}\right) V_{1}}{\rho\left(1-\zeta_{2}\right) V_{2}}+\frac{\rho\left(1-\zeta_{2}\right) V_{2}}{\rho\left(1-\zeta_{1}\right) V_{1}}\right] .
$$

In the above equations, $\Omega$ denotes the phonon frequency, $V_{1}$ and $V_{2}$ are the constituent layer phonon velocities of the given mode (transverse or longitudinal), $\zeta_{1}$ and $\zeta_{2}$ are the constituent layer porosities, and $\rho=2330 \mathrm{~kg} / \mathrm{m}^{3}$ is the density of bulk crystalline silicon.

With the optical and elastic parameters of the constituent layers known, the theoretically expected dependence of the phonon frequency on $D$ (for a given constituent layer thickness ratio) was obtained. This was done for each set of SLs by numerically determining the values of frequency that satisfy Eq. (3) for given values of $D$. The resulting curves are shown in Fig. 3 along with experimental data obtained directly from the Brillouin spectra. The agreement between the data and the curves corresponding to the folded longitudinal acoustic (FLA) modes indicates that zone-folding of the longitudinal phonon has occurred. Moreover, comparison of Figs. 3(a) and 3(b) suggests that small differences in the constituent layer parameters manifest as observable changes in the behavior of the FLA modes. It is noted that the relative uncertainty in $D$ was estimated as the $\sim 10 \%$ difference between measured SL film thickness and that calculated using etch rates, etch times, and number of current alternations. The error in measured phonon frequency was taken to be the 
standard error in the Lorentzian fits of the Brillouin peaks or, in the case of weak features, simply estimated directly from the spectra. There is also $\sim 10 \%$ uncertainty in the calculated frequencies due to uncertainties in the measured single layer film porosities, refractive indices, and acoustic velocities which serve as input for the SL model calculations.

Observation of Brillouin zone-folding suggests that these $\pi$-Si SLs may behave as hypersonic phononic crystals. In

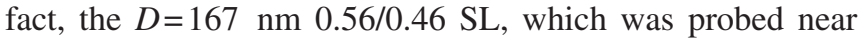
the edge of the third phononic Brillouin zone $(q D \approx 3 \pi)$, contains a spectral peak doublet that is the manifestation of a hypersonic phononic bandgap in the longitudinal bulk phonon frequency spectrum [see Fig. 2(d)]. The two peaks (1 and 2), which were assigned to two adjacent FLA branches [see Fig. 3(b)], had frequency shifts of 11.8 and $15.1 \mathrm{GHz}$, corresponding to a gap of width $3.3 \mathrm{GHz}$. This is in reasonable agreement with the calculated gap, which ranges over $11.8-13.9 \mathrm{GHz}(2.1 \mathrm{GHz}$ width). Also, a third peak (3) was observed and it corresponded to the edge of the second phononic bandgap, which was expected at 37.5-39.5 $\mathrm{GHz}(2.0 \mathrm{GHz}$ width). The absence of a peak doublet at these shifts may be due to phonon attenuation, an effect which is not accounted for in the current calculations. Attenuation may cause the expected gaps to shrink or even disappear altogether, resulting in a single spectral peak instead of the doublet predicted by the present model. ${ }^{4,5}$

From Fig. 3 it can also be seen that the data not attributed to the FLA modes agree only qualitatively with the calculations for the folded transverse acoustic (FTA) phonons. This may be because the corresponding spectral peaks are actually due to some unidentified mode rather than FTA phonons and thus the current theory might not apply. Transverse phonons, compared to longitudinal phonons, typically have a small scattering cross-section in backscattering geometry and hence it is possible that the FTA modes were not seen in the SL spectra.

In the region $2 \pi \leq q D \leq 3 \pi$ (between the second and third Brillouin zone edges) one can see that, with the exception of the $D=167 \mathrm{~nm} 0.56 / 0.46 \mathrm{SL}$, none of the data points show strong agreement with the second FLA curve. It is conceivable that this mode was too weak to be observed and the data at $\sim 18 \mathrm{GHz}$, despite showing some agreement with the curve in question, may actually be due to some other mode. Theoretically, the intensities of the zone-folded modes in a SL with perfectly planar layer interfaces vary dramatically from branch to branch and are expected to be dependent on $q D$ (changing drastically near a zone edge). ${ }^{3,5}$ The peak intensities are further affected by the presence of diffuse layer interfaces ${ }^{6,7}$ and optical-absorption, ${ }^{5}$ as may be the case with these $\pi$-Si SLs. These effects could lead to missing FLA orders which are only readily observed for certain values of $q D$.

Finally, according to Eq. (2), these SLs should be onedimensional photonic crystals. ${ }^{8}$ Preliminary results suggest that this is the case. In particular, SLs with values of $D$ in or near the shaded region of Fig. 3 are green when viewed at normal incidence in ambient light, while those with $D$ above or below this region appear red or violet, respectively. Work is ongoing to determine how the photonic nature of the SL affects the Brillouin spectrum.

In summary, Brillouin scattering experiments, coupled with calculations based on a one-dimensional elastic continuum model, have revealed the existence of zone-folded longitudinal acoustic phonons and a hypersonic phononic bandgap in porous silicon superlattices.

This work was supported by NSERC Grant No. RGPN261546 and the Canada Foundation for Innovation. The authors would like to thank A. Polomska for helpful discussions.

${ }^{1}$ C. Pickering, M. I. J. Beale, D. J. Robbins, D. J. Pearson, and R. Greef, J. Phys. C 17, 6535 (1984).

${ }^{2}$ G. T. Andrews, A. M. Polomska, E. Vazsonyi, and J. Volk, Phys. Status Solidi A 204, 1372 (2007).

${ }^{3}$ J. He, B. Djafari-Rouhani, and J. Sapriel, Phys. Rev. B 37, 4086 (1988).

${ }^{4}$ G. Aliev and P. Snow, Mater. Res. Soc. Symp. Proc. 1145, MM13-04 (2009).

${ }^{5}$ J. He, J. Sapriel, and R. Azoulay, Phys. Rev. B 40, 1121 (1989).

${ }^{6}$ C. E. Bottani and R. Caporali, J. Phys.: Condens. Matter 9, 5787 (1997).

${ }^{7}$ C. Colvard, T. A. Gant, M. V. Klein, R. Merlin, R. Fischer, H. Morkoc, and A. Gossard, Phys. Rev. B 31, 2080 (1985).

${ }^{8}$ V. Agarwal and J. A. del Rio, Appl. Phys. Lett. 82, 1512 (2003). 
Applied Physics Letters is copyrighted by the American Institute of Physics (AIP). Redistribution of journal material is subject to the AIP online journal license and/or AIP copyright. For more information, see http://ojps.aip.org/aplo/aplcr.jsp 